\title{
"They are like personalized mini-Googles": Seeking Information on Facebook Groups
}

\author{
Preeti Mudliar \\ IIIT-Bangalore \\ preeti.mudliar@iiitb.ac.in
}

\author{
Noopur Raval \\ University of California Irvine \\ naraval@uci.edu
}

\begin{abstract}
Our study reports on everyday life information search (ELIS) practice using Facebook Groups. While previous research has examined social $Q \& A$ in the context of status message question asking (SMQA) on Facebook and Twitter, we discuss how people step outside their personal networks to find answers to questions while staying within the Facebook environment. We investigate two popular Q\&A Facebook Groups in the city of Bangalore, India and ask why people turn to Facebook Groups for the information needs, the nature of costs of using these groups for information search, and how Groups are groomed to host social Q\&A practices. Our findings suggest that Facebook Groups can be popular venues for information search because of its structural features as well as the networked sociality that it engenders.
\end{abstract}

\section{Introduction}

As alternatives to search engines, platforms and processes that support social search behavior, have received considerable research attention in the recent past. Notable instances include social Q\&A sites such as Yahoo! Answers, a social search engine like Aardvark, and knowledge sharing sites that allow users to follow each other such as Quora. In addition to this, SNS platforms such as Facebook and Twitter have also been examined for their potential to support information search through status message question asking (SMQA) e.g. [1, 12, 18, 19, 22, 23, 29, 36, 37, 38, 41, 43].

In this paper, we extend the research on social media information search to draw attention to search processes performed using SNS features other than status updates on personal profiles. We direct our efforts specifically towards two Facebook Groups that have organically emerged as active and vibrant hubs of rich interactions around information needs. Formed and groomed by owners, moderators and members alike, these groups act as spaces where people who are not necessarily a part of each other's personal network on Facebook post questions to solicit and engage in collaborative search for their everyday life information needs from group members. Thus, they emerge as online social Q\&A spaces even while they are embedded in the Facebook environment. Jones and Rafaeli [24] introduced the term, 'virtual publics' and described them as "symbolically delineated, computer-mediated space that enable a potentially wide range of individuals to attend and contribute to a shared set of computer-mediated interpersonal interactions" (p. 216). We found this definition typical of the nature of Facebook Groups in our study. The spaces that we report on are composed of a large number of users who usually have no pre-existing ties with a majority of the other members and are largely unknown to each other. The interactions on these groups are characteristic of Wittel's [52] notion of network sociality that consist of "fleeting and transient, yet iterative social relations; of ephemeral, yet intense social encounters" (p.51) that seek to resolve questions through online conversations and interactions in the form of comments on a group post.

Similar to online social Q\&A sites, the content and kind of questions asked in these groups are typically wide ranging [1]. Questions and responses are persistent rather than ephemeral and archived for future consumption allowing members to 'listen' in to conversations. Since Groups also have a search bar, it enables members to search the archives for posts. This makes Groups supportive of both implicit and explicit searching behavior that is also collaborative in nature [16, 34]. Lastly, the nature of Facebook's News Feed, which streams content on user profiles, ensures that members receive group content depending on the algorithmic calculations specific to their profile without having to always actively browse through the group.

\section{Facebook Groups}

While Facebook Groups have been the subject of some research interest, specifically as venues for information sharing and community building around specific causes, there have been no systematic attempts to explore how the Facebook Group environment supports information search $[5,25,36]$. At least three studies have adopted a 'Uses \& Gratifications' approach to understand how and why people 
use Groups. Though the investigation contexts of these studies differed, the studies found that membership and participation in Groups fulfill needs such as expressive information sharing, entertainment, socializing, social interaction, contribution, discovery, self-status seeking, and information [25, 37, 43]. Recent research on social media use among specific populations such as parents of children with special needs have also revealed that Groups are one of the online resources that provide "networked empowerment" to parents by facilitating access to information, social support, and advocacy [2, 3]. However, the primary emphasis of these studies remains on the users and their need fulfilment for which they seek and build online communities.

In our paper, we contribute to existing literature on social media information search and Facebook Groups in three ways. First, we direct attention to the experiences of people who even while remaining within Facebook, reach outside their personal networks to engage in what Savolainen [39] proposes are everyday life information search (ELIS) needs instead of confining themselves to search via SMQA. We detail the situations and contexts of information search that make Groups (as against personal updates and search engines) are particularly desirable venues for the respondents. Secondly, we trace how the inherently social nature of information-seeking unfolds within these groups. We offer an understanding of how social search in this context surfaces underlying tensions of both expertise (regarding a city, traditions, motherhood, religion and more) as well as moralities that can sometimes lead to trolling and cyberbullying. Finally, we present how people engage with the design and platform affordances of Facebook Groups to negotiate the intersecting politics of social search in a human-machine environment.

\section{Related Work}

\subsection{Social Search}

The experience of asking questions on SNS is significantly different from online social Q\&A sites such as Yahoo! Answers and Quora, owing to different socio-technical features. While social Q\&A sites afford users the choice of anonymity or the use of a pseudonym, SNS are associated with real names and identities which correspond with higher levels of trust and reliability of information. As compared to social Q\&A sites, SNS also afford information seekers a more niche and limited audience as it is confined to their personal network along with restrictions on character length of status updates $[18,27,28,49,50]$.

From their study, Morris et al [36] determined that almost $50 \%$ of Facebook and Twitter users had turned to their networks on these SNS to search for information by broadcasting requests for help using their status messages. Hirsch et al [18] found that people used SMQA for almost
$20 \%$ of their information needs in comparison to search engine use. People turned to SMQA for answers when they needed responses that were tailored to their needs and they believed that their personal network was both trustworthy and aware of their preferences. Also, SNS facilitated ease of contact and by virtue of social connections assured better speed and answer quality for non-urgent tasks. Compared to search engines, Hirsch et al found that SMQA was deemed more satisfactory because it fulfilled social needs [18]. For instance, though question askers found information from close ties less useful, they also rated it highly satisfying owing to the social engagement with ties.

Most of the research on SNS information-seeking has examined search behavior in the context of personal networks on SNS and by using quantitative methods including surveys, laboratory studies, publicly available SMQA updates, and server use logs to study motivations for SMQA use, the content matter of questions, patterns of responses, and the quality and satisfaction that people derived. While asking strangers for information on SNS has been explored, it has been investigated in the context of Twitter which is a structurally different environment than Facebook [38]. We argue that Facebook Groups offer a unique group setting for interaction among strangers with rich real social identities and hence merit investigation for how they facilitate search among relative strangers. We adopt a qualitative approach to locate the experiences of people using two such sizable groups as information search resources and the value that they derive from it.

\subsection{ELIS on Facebook Groups}

We find the ELIS framework as proposed by Savolainen [39] particularly valuable for this study as it recognizes that information seeking is not limited to purposive goals in solving a problem situation or restricted to workplace activities. Instead, as Savolainen states, people are engaged in information-seeking behaviors that are closely connected to their routine everyday activities as a natural extension of their everyday practice. Savolainen thus defines ELIS as information elements that people use in their everyday life and resolve problems that are not always connected to their profession, but span across different areas of routine life. He also frames information seeking as a process that seeks to achieve "mastery of life" (p.272) through a passive monitoring of everyday life events and argues that it is a continual lifelong activity that is integral to how people navigate everyday life matters.

This passive monitoring of information segues into an active information search only when people are confronted by a problem that disturbs their routine order. Hence, not all information is gathered through an active systematic search, but a significant part of information that is meaningful to our daily lives emerges just by way of life experiences. Smock et al [42] argue that it is useful to think of the Facebook 
environment as a "collection of tools" (p.232) that are used in different ways to meet different needs because different features engender different communication behaviors. In the context of Facebook Groups in particular, they note that the one-to-many broadcast communication feature of Groups motivates expressive information sharing to a group of strangers with like-minded interests.

\subsection{Costs and considerations in SNS Information Search}

While personal networks on social media are emerging as a handy and complementary alternative to search engine information search, people often avoid turning to their personal networks for help due to multiple reasons such as viewing SMQA as unsuitable for deeply personal topics like finance, health and dating. They also censor their broadcasts because of sensitivity to certain individuals' presence in their network [32, 45]. People also refrain from asking questions through status updates if their information needs are too specific or they are unsure about what they are looking for [18]. Participants also consider that their personal networks might not know enough about the topic or that their low 'Friend' count might limit the search [47]. Similarly, people avoid posting controversial questions for they might disrupt interpersonal relationships because maintaining sociality and information seeking are both crucial considerations in SMQA. In that sense, people are also mindful of aligning their queries to match how they are perceived socially online $[18,50]$.

In terms of motivations to participate in SMQA (both asking and responding), Facebook has been identified as the means to generate social capital where both asking questions and responding or helping out appear as a form of sociality, to reach out to Friends of Friends (or weak ties) [17]. To harness the social resources available through weak ties, people often engage in relationship maintenance activities [12]. Research on FRMB (Facebook Relationship Maintenance Behaviors) reveals that people engage in various communication patterns for relationship maintenance [12]. For instance, 'signaling' strength and context based on content, frequency, and message length [11], expressing curiosity about others and desire to stay in touch [48], engaging in public comment exchanges [53]. It is now known that the "grooming" labor spent in cultivating and maintaining one's social network affects the quantity and quality of responses to one's request for help [28]. The studies mentioned above explore grooming practices within interpersonal relationships. Our paper turns this lens on Facebook Groups to discuss social capital accrual by detailing strategic individual decisions around time, tone, gender, topics and more in these socio-informational exchanges.
Through a detailed investigation of two groups (B1 and B2), we ask:

1. Why do people turn to Facebook Groups for their ELIS needs?

2. What are the qualitative features of Facebook Groups such as B1 and B2 that facilitate the process of social search

3. How do people strategize and negotiate with the technosocial platform politics of Facebook Groups in their social search quests?

\section{Methods}

Our study focuses on two closed Facebook Groups - B1 and B2 (anonymized) predominantly composed of residents of Bangalore, chosen based on their popularity as resources for information seeking. Both groups have a sizable number of members and have been repeatedly covered in local TV news, print and radio shows [45, 46]. B1 shot to limelight following the media coverage of an incident in September 2014 when the group's collective action (through a Lost and Found announcement and responses) helped return a lost wallet to its owner. The incident was also featured on Facebook's official page [14]. B1's success led to numerous impersonators who appropriate its name and purpose to own their separate B1 spin off groups in different cities and even neighborhoods in Bangalore (over 60 such spin offs exist at the time of writing). Similarly, B2 was recently featured as a popular "round-the-clock service" [45] for mothers and for women at large as a resource for various questions.

B1 was formed in May 2011 by the second author to facilitate storing information about Bangalore (accommodation, travel, education, food queries) permanently and sharing it with her acquaintances and others beyond her personal network. Currently it has over 100,000 members and three administrators. Facebook Groups provided a relatively stable and semi-public (friends of friends) platform for the founder to share and archive such information so that she wouldn't have to repeat it individually in the future.

B2 is a women's only group that was formed in February 2012 by a British woman who is married to an Indian. The group came into being to address the founder's own personal need of finding an easy way to coordinate playdates for her two children with her existing group of other mum friends in the city. Over time, it grew to be socially co-opted by its members as an information and support seeking resource. Though it is composed predominantly of mothers living in Bangalore, membership is inclusive to all women. At the time of this writing B2 had 26,500 members and is solely moderated by the owner. While both groups began as close networks for friends of the founders, over time they grew to become "public" (where most members had no personal connections to founders). We chose to study two groups to mitigate any biases that could have colored our study of the 
groups owing to the second author's personal investment as the founder and owner of B1. The study thus leverages the first author's neutral relation and membership to both the Groups along with the second author's situated knowledge [30] of B1 as a Group owner.

The paper is informed by 50 semi-structured interviews with members, former members, and administrators of the groups. It is complemented by both the authors' observations as long-standing members of B1 and more recently B2 and also the second author's role in founding and moderating B1. The first author has been a member of B1 since June 2013 when she moved to Bangalore and a member of B2 since February 2015. Though the second author continues to retain ownership and moderator status of her group, she had ceased active moderation since 2013 and had delegated additional moderation to four other members. She joined B2 in June 2015 after the group owner consented to allow the authors to include the group as a study site and reach out to members for interviews. Both the authors are women which allowed them to become members of $\mathrm{B} 2$.

\subsection{Data collection and Analysis}

Participants were initially recruited through purposive sampling, guided by the authors' observations and familiarity with members on the groups. Interviews lasted between 45 to 90 minutes. We asked participants how they learnt of the groups and to describe their use of the group in terms of asking questions, commenting on other's questions, discussions and conflicts with other members, communication with moderators, forging connections with members on the groups, how they managed their privacy on the groups, and the critiques they had about the groups.

As interviews progressed, participants volunteered additional names resulting in snowball sampling. Our research design was emergent [10] and remained alert to the diverse ways in which members were experiencing the groups. We were guided by the narratives that participants shared with us allowing us to locate and interview members who were active, former members who left the group or had been banned by moderators, members who made offline connections through interactions with other members on the group, members who appropriated the group for enterprise, and members who experienced trolling and conflicts with other members on the group.

Recruitment and interviews were conducted over a period of three months from June-August 2015 during which we contacted a total of 67 people across both groups. Many of our participants had overlapping memberships in both the groups that we were studying as well as other niche special interest closed and secret groups around themes ranging from real estate, food, and a secret sisterhood group for women. Hence, we also interviewed owners and moderators of such groups because they repeatedly emerged as frequently mentioned groups of relevance to our participants. Off the 67 members that we contacted, $50(n=50)$ members responded and consented to be interviewed for the study. 27 interviews were conducted in face-to-face settings and 23 interviews were conducted over the phone. Two interviews were conducted over email and chat due to location and privacy constraints of the participants. Participants were offered gift vouchers worth INR 500 [approximately US \$7] as a token of appreciation for their participation.

Once consent on studying both the groups was achieved, the authors began contacting group members for interviews. To avoid respondent bias of $\mathrm{B} 1$ members towards the second author's founder status, most B1 members were interviewed by the first author and most B2 members were interviewed by the second author. Although B1 is not limited to Facebook users from Bangalore, given that its inception and initial seeding of members from the founder's own social network involved adding friends and acquaintances from Bangalore, the membership and content in the group is predominantly Bangalore specific. Similarly, while B2 largely caters to moms in Bangalore, it still has members who have migrated to other cities but continue with their membership on the group and members who live in other cities, but have joined the group through friends' recommendations.

Based on demographic data collected through a survey, on an average, our participants were 31 years old (age range 2455 ) and had lived in Bangalore for six years (residency range 1-more than 6 years) while 5 participants were native residents of the city. In terms of 'search-use', top platforms reported were search engines and Facebook Groups (73\%), own Facebook profile (60\%), WhatsApp groups $(30 \%)$, Quora (13\%), and Twitter and LinkedIn (1\%).

After transcription, we applied the principles of iterative pattern coding and constant comparative analysis [15] and each author engaged in a line-by-line reading of every interview transcript multiple times to trace emergent codes and categories from the data. We were guided by the principles of grounded theory [44] in our analysis. Both authors coded the transcripts independently at first and later exchanged theoretical memos and themes that emerged from their coding. These themes were discussed by the authors in line with related literature to form categories. The categories were captured on spreadsheets to enable joint tracking and iterative analysis until we arrived at the categories that we use to report the findings in this paper.

\section{Findings}

\subsection{Why Facebook Groups?}

Most participants we interviewed pointed to the existing ubiquity of Facebook use in their daily life as a reason for the Groups' preference as venues for information search. We 
frequently heard expressions attesting to the popularity of Facebook, "everybody is on it!" For participants, the Facebook environment offered a "place" to integrate and bifurcate their overall social network use and specific use. We discuss some of the reasons that participants offered for turning to groups for information:

\subsubsection{People help you search through scenarios}

Participants often reflected on their information search on Groups by contrasting it with their growing years when strong social ties between neighbors and communities meant that "real people" were always at hand to answer ELIS questions. They expressed that with rapid urbanization and migration, the traditional way of information-seeking that involved reaching out to strong social ties in their current place of residence was not always readily available. The common primary motivation that participants cited for using Groups such as BI and B2 was that these spaces allowed their queries to be answered publicly by "real" people while engaging in discussions around their questions. One member of B1 who also moderates a popular Bangalore real estate Group explained, "It is people. Give me a better platform to connect with people. Don't think so. Facebook is all pervasive and has a wide reach."

A member who had used B1 to find a late-night restaurant said, "Search engines only give you a bunch of algorithmically determined links. If I am looking at Zomato (restaurant search platform), I have reviews, but little context as to who they are from, but on groups like B1, I can reach a very large sample of real people who tell me things based on their own experience. I can even follow up with them through comments and messages on Facebook if I need more clarifications."

Yet, another participant who managed to buy the same shoes that her friend had purchased in another city by querying B1 members added, "Imagine if you had people helping you out, every time you had a query. That is what makes these groups work."

Simultaneously, as a participant indicated, information search through "real" people in "real-time" is more reliable than Google search because the latter can have outdated information. However, Groups such as B1 and B2 helpfully provide temporal and other caveats to queries. For instance, if looking for a particular shop, responses such as "might not be open anymore", "relocated to another venue" were helpfully crowdsourced through discussions on Groups. Participants pointed that the most current information along with tangential context and first-hand reviews would be very difficult to find on search engines.

Members also reported information found through Group queries as "not paid" or without "commercial motives". While there are business promoters advertising on Groups, members reported that it was easy to discern "genuine content" from the rest. Members also pointed out that
Facebook's presence as a "large content database cannot be matched by one's personal Facebook network". Other participants pointed to the real time and quick feedback they could expect by posting on groups with a large engaged member size that was not possible with SMQA

5.1.2. Online Social Interactions and Offline Connections Across our interviews, we found that members cherished the interspersed interactivity and sociality within each Group conversation they participated in. To illustrate, a participant was looking for movie recommendations, but could not name the genre and when he put up his question, he was unsure what he should be asking and the members on his group helped craft his question. He said,

"I like watching certain kinds of movies, but I did not know how to describe them. So, I used examples and asked people to recommend similar films since neither Google nor IMDB were helpful. But on the group, people pitched in to help articulate what I was looking for. I could also discuss elements of the films that they were suggesting which was so much fun and useful."

Another participant who was well known on B1 for his humorous banter on posts said, "The whole aspect of Groups on Facebook is social interaction and if you take human interaction out of the equation then you just have a question answer thing which we have plenty of." Hence, the inherently convivial life of information (seeking and providing) is central to the "stickiness" of Group Q\&A.

Affect marks interactions in several ways on Groups, not only in positive ways. Reputation-building is one such marker where participants are conscious how the nature of their query and the utility of their responses would affect their reputation and they also reported calling other members as "witty", "knowledgeable", "helpful" and "sanctimonious". Simultaneously, information-seeking also operates as a mode of social interaction where we observed people forging offline friendships that started because of conversations on Groups.

For instance, describing the connections, she formed through, one participant who was active on Bangalore-based food, second's sale, and travel groups in addition to B1 and B2 said, "I have about 30 friends in my life that I have met through these groups due to interactions over questions. Some of them are very close to me. Questions generate conversations and you know of people who regularly respond and you find people with whom you really click."

\subsection{Qualitative Features supporting social search}

\subsubsection{Hyperlocal Hybrid Spaces}

Given their emergence at the intersection of online search and offline sociality, we found the interactions on B1 and B2 deeply entangled with social and physical geographies and reflecting what Taylor et al call "data-in-place" [47]. Both B1 and B2 produced a semblance of geographical and socio- 
political boundaries as "virtually local" [26] spaces. While B1 carried no mention or description of its place of conception (the founder was in Bangalore when she started it), B2 explicitly identified as local (it had Bangalore in its name). However, both groups maintained 1) a strong local focus and 2) went on to become national phenomena through clone groups and local chapters.

We found that the "local" had different but important implications in how the groups evolved. B1 remained intensely local (Bangalore-centric in content) despite there being members from all over India and its founder having moved to a different city. Not only this, as several members shared in their responses, B1 catered to a limited, specific demographic within Bangalore, especially young, highskilled immigrant IT workers; whose presence, Internet use, information and resource needs and affordances have significantly shaped economic activity in Bangalore in the past two decades [45].

Members on B1 who identified as 'local Bangaloreans' (referring to those born and raised in the city) often mentioned in their interviews how the activity on the group reflected the needs of immigrants who had no knowledge of Bangalore's neighborhoods, local joints, parks and resources. On the other hand, B2, which also explicitly included 'Bangalore' in its title had a variety of ways in which the 'local' character of interactions unfolded owing to the nature of discussions, which largely centred around issues pertaining to women and children. Here, the local manifested in the form of cultural traditions of child upbringing, rituals of married women and assertion of local linguistic identity (Kannada). Older women also found themselves advising younger women to navigate concerns ranging from childcare to domestic violence in the absence of traditional familial social ties. For instance, an older mom remarked in her interview about younger moms' queries:

"These younger moms don't have the support of their mothers anymore. They come from small towns to Bangalore, they are mostly alone, don't know where to get advice. So, they read books and download apps and keep posting on the group."

5.2.2. Localized flavors and contexts of information Members of both B1 and B2 often remarked on the ways in which B2 rooted them to the city. Important also, is the fact that such local data is translated for use by members for people unfamiliar with local contexts of Bangalore city for queries required this translation thus making "those in similar situations" a searchable category. These highly situated uses not only grounded the online space in the physical and socio-political space of the city, but also simultaneously anchored the space of the online group within the boundaries of Bangalore through their content. As a stay-at-home mother who was a regular poster on B2 expressed, "It's like a personalized mini-Google because it customizes the search experience for you in the context of your needs and you can also search the archives."

In our supplementary interviews with owners of mom Facebook Groups in the cities of Mumbai and Chennai, the founders delineated the "cultural differences" between the groups. The Mumbai group founder emphasized how her group was for "happy conversations" since she felt that exchanges in the Bangalore group sometimes took dramatic and unpleasant turns. The Chennai group owner admitted that while her inspiration was the B2, her own group strictly discussed mothers' utility topics. In that sense, ELIS exchanges on these groups were not only about exchanging helpful experiential knowledge within the extended social network but they also took on discursive flavors where some queries were reflective of certain identities and responses were imbued with cultural, moral and political judgment. Examining the responses of participants, we found that while both the groups were indeed valuable as convening sites for real people, 'people' here function as more than factproviders - they appear as social agents with shared experiences, queries and anxieties by virtue of sharing an urban space and embodying common identities such as Bangalorean, mother, immigrant, Bengali etc. thus, representing the informational interests of a larger passive mass that is reading Group Q\&A.

Participants frequently expressed that Group Q\&A threads reflect typical information situations that "people like me" find themselves in, also implying how Group Q\&A threads gathered information in specific temporal and situated ways for retrospective and tangential use.

\subsection{Facebook Groups as an Information Platform}

Both the online information exchange relationships and the offline social networking it sometimes engendered, must also be situated as specific byproducts of Facebook's platform features. Here we present observations on platform affordances and design and how they shape and support information search within Groups For instance, an active commenter on B2 group recounted that instead of reading or watching TV (her earlier hobbies), she now prefers logging on to B2 and "keeps scrolling down" as she learns "interesting" and "relevant" information there.

\subsubsection{Platform Conveniences}

Similarly, we heard many participants describe Facebook Groups as "convenient" and as we unpack the term, it is obvious that Facebook doubles up as a social network and information sharing platform largely due to its dominance as a platform that everyone is 'always on.' As participants recalled, being able to access so much information "within the same tab, without having to type an additional username and password elsewhere" speaks of how Group members 
view Facebook as an integral part of their routine virtual hang-out venues to which they are always logged in.

It is worth mentioning that we unearthed several instances where Group owners and moderators tried to build standalone websites to monetize their Groups' success but mostly failed (barring B2 which was still work in progress). Members expressed that visiting a website "only to answer questions or help others" or even sell and buy goods was too much effort, signaling to the comfort and familiarity of a Facebook-structured social world as a backdrop that sustained transactions and interactions in Groups.

The passivity of receiving information without "having to do anything" received frequent mention from participants who pointed both to the nature of the news feed that pushed content on their timeline to being 'readers' of the group. It also illustrated Crawford's [8, 9] notion of lurking as a form of active participation. Some participants shared how when they first joined the group, they 'listened' by keeping up with the conversations. While some preferred to actively spend time scrolling through the group feed to read the questions and interactions, others said that they had edited their News Feed to prioritize the streaming of group content because receiving information was often happenstance, accidental, and serendipitous. One participant confessed to have unfollowed his friends in order to receive more content from his Facebook groups including B1.

\subsubsection{Politics of Visibility}

The size of the groups, during their respective lifecycles also produced novel opportunities and constraints in terms of the quality of engagement, effects of relative anonymity and context collapse. We describe here what we dub as the "costs" of information search tied directly to members' social engagement on Groups. A respondent who had been an 'early member' of both B1 and B2 in 2012 (when the groups had about 2000 members), correlated the rise in harassment and unwanted messages to the growth in membership. Since B1 had started out as a 'weak ties' model (friends of friends) and the ties got looser with its growth, and since Facebook provided no additional support as a group grew, posts with the latest comments automatically appeared at the top, pushing posts that did not receive a quick response at the bottom to relative obscurity.

Members worked around this loss of visibility by commenting on their own posts, by simply writing "bump" to bump the post up in the feed. These creative workarounds that sought to strategize the Facebook Groups design and affordances to the benefit of members led to their own politics of visibility. Members reported losing interest, losing faith, and getting annoyed with others who could afford to spend more time doing "gimmicky things" to gain visibility. As one member narrated, “...now when you post, either you don't get a response because of high traffic or you need to be a girl with a pretty picture or your question has to be interesting to get attention so I only use the group to search its archives." As is known in other studies of communities, participants who did not receive answers to questions reduced their participation and developed negative feelings towards the group [5].

On the flipside, increased visibility had its own consequences. Members reported having become Facebook friends and sometimes even offline friends based on their group interactions with other members. Some members reported having brought their family, friends and acquaintances on to the group because of its utility. In both cases, the presence of stronger ties led to more selfconsciousness and often also self-censorship. Participants reported being careful while asking for travel recommendations because they did not want their colleagues to know that they were planning trips. Similarly, in the women-only group, members reported avoiding posts about personal issues, health and marriage because they knew that their neighbors, relatives and friends might see it. Both these issues (of invisibility and contextual privacy**) point to the nature of Facebook Groups as a unique hybrid space that in some sense allowed members to selectively perform (reveal, hide, rearticulate) their identities, in-turn providing a space where they could (or later could not) ask and share information that might be atypical of them in their personal, professional and social networks.

Importantly and predictably, there were more serious repercussions to increased visibility such as cyberbullying, trolling, moralizing and harassment. There were parallel discussions on keeping information exchange as a valueneutral and apolitical activity as well as on "moderating" or regulating what kind of questions and answers were permissible, arguing that the content of the group shaped the very "nature" of the space. One such recurrent topic on B1 included posts offering or requesting for dogs of certain breeds on sale. Discussions on these posts quickly devolved to shaming, name-calling and chastising members interested in selling or buying dogs as opposed to adopting strays. Similar controversial topics on B2 included anything pertaining to children (advice on schooling, disciplining, nutrition, daycare) where some members would inevitably question the very morality or parenting skills of the requester. One mom (member) reported how the vicious and hurtful comments on her post requesting daycare information, while she was already battling Postpartum Depression made her swear off posting on the group forever.

Some ways in which members toed the fine line between invisibility and hypervisibility included getting friends to post on their behalf. The owner and moderator of B2 also offered to regularly put up posts marked 'anonymous' that she received from members who did not wish to disclose their identity for reasons of privacy and trolling concerns. 
This 'service' on B2 is highly popular and many members use it to ask for help on matters ranging from domestic violence, coping with mental health concerns, resolving issues with in-laws, and sexual and reproductive health questions.

Another tactical way in which members tried to attain "maximum positive visibility" (where their post gets maximum attention but is also resolved before trolls descend) was to figure out best posting timings. Like a member said, "I have found Friday evenings and weekends poor times to post, but if I post something on Tuesdays or Wednesdays, the responses flood." Some members also deleted their posts once they got the answers.

\section{Discussion}

Our study sought to broadly understand the reasons that made Facebook Groups useful for people to share knowledge and engage in ELIS needs through active and collaborative information seeking, passive monitoring of information, and archival search. We were motivated to understand and detail how the habitual and everyday sociality of Facebook is intertwined with the everyday routines of people to constitute active and passive information search activities on Groups. While SMQA behaviors have been studied in the context of Facebook, we explored how Groups owing to their technical affordances that are embedded in the larger Facebook environment can also be socially harnessed to serve a constant stream of information flow for its members.

In the context of past research on SMQA, we found that customized responses to questions and trust in information were not necessarily restricted to personal SNS networks. Instead, we found that because Facebook allowed people to discern in some measure the authenticity of others, participants were willing to broadcast their questions and interact with strangers in favor of reaching a wider audience and thus harnessing the strength of weak ties even while seeking enjoyment and satisfaction as active seekers and passive monitors of information. The hyperlocal nature of questions and responses created a mesh of knowledge and social connections - both online and offline that were constitutive of and enabled the organic construction of a crowdsourced and peer approved digital urban infrastructure for a city. As research attests, people often repurpose tools that are part of their everyday routine for information search, so collaborative tools that are lightweight and form a "glue" system with their existing social and information ecosystem would likely be more favorably received by users [34].

Given the nature and characteristics of information search in a collaborative group setting, this study also reveals that other than issues of privacy and self-presentation that also occur in SMQA, costs incurred in looking for information in groups have to contend with cyberbullying in the form of moralizing and trolling since questions often reveal values and choices.

In the quest to build better search systems and social Q\&A sites that will motivate users to share accurate, relevant, and contextual information with each other, researchers and designers have explored ways to design socially supported search including forms of friendsourcing and reaching out to strangers with targeted questions [5, 13, 34, 35]. We hope that our investigation of Facebook Groups demonstrates the efficacy of reaching out to people who co-inhabit the same socio-technical environment and also linger around to intercept information passively, provides directions to efforts to make sharing information socially enjoyable while providing for ways to minimize social costs.

\section{Limitations and Future Directions}

Our study is based on qualitative observations and interviews with a limited number of members of two Facebook Groups with a predominantly Bangalore based population to understand their experiences of information search. Groups are a feature of Facebook and their purpose is socially determined leading to a variety of agendas. As such, we make no claims to the purpose, and experiences of members of other Facebook Groups or even all members of the two groups that we studied. Our sample may have also reflected the experiences of more active group members though we made concerted efforts to search and include currently nonactive and passive members.

In view of extant research on SMQA and online social Q\&A sites, future research directions for understanding social Q\&A on Facebook Groups would include studying the content and type of questions posted on the group, time of posting that receive more responses, comparing satisfaction and routing of information needs between search engines, SMQA, and Groups and the network size and composition of groups. Since the groups that we studied were also closed, privacy concerns and easy identification of members with their posts and comments can limit the kind of data that can be accessed. Public Facebook Groups of a similar nature can perhaps broaden the kind of data available for analysis.

\section{Conclusion}

In this paper, we directed attention to Facebook Groups as a socio-technical environment for fulfilling active and passive ELIS needs. Our exploration specifically highlights the ways in which people balance their 'real-world identities' and information needs. Our qualitative examination of two Facebook groups brought to light a new venue where people engage in information search. We hope our analysis and discussion of why and how Groups fulfill information needs will lead to newer ideas and explorations in social information search behavior. 


\section{References}

1. Lada A. Adamic, Jun Zhang, Eytan Bakshy, and Mark S. Ackerman. 2008. Knowledge sharing and yahoo answers: everyone knows something. In Proc. of the 17th international conf on World Wide Web (WWW '08). ACM, New York, NY, USA, 665-674.

http://doi.acm.org/10.1145/1367497.1367587

2. Tawfiq Ammari, Meredith Ringel Morris, and Sarita Yardi Schoenebeck. 2014. Accessing social support and overcoming judgment on social media among parents of children with special needs. In Proc. of the International Conf on Web and Social Media (ICWSM'14).

3. Tawfiq Ammari and Sarita Schoenebeck. 2015. Understanding and Supporting Fathers and Fatherhood on Social Media Sites. In Proc. of the 33rd Annual ACM Conf on Human Factors in Computing Systems (CHI '15). ACM, New York, NY, USA, 1905-1914.

http://doi.acm.org/10.1145/2702123.2702205

4. Jaime Arguello, Brian S. Butler, Elisabeth Joyce, Robert Kraut, Kimberly S. Ling, Carolyn Rosé, and Xiaoqing Wang. 2006. Talk to me: foundations for successful individualgroup interactions in online communities. In Proc. of the SIGCHI Conf on Human Factors in Computing Systems (CHI '06), Rebecca Grinter, Thomas Rodden, Paul Aoki, Ed Cutrell, Robin Jeffries, and Gary Olson (Eds.). ACM, New York, NY, USA, 959-968. http://doi.acm.org/10.1145/1124772.1124916

5. Michael S. Bernstein, Desney Tan, Greg Smith, Mary Czerwinski, and Eric Horvitz. 2008. Personalization via friendsourcing. ACM Trans. Comput.-Hum. Interact. 17, 2, Article 6 (May 2008), 28 pages. DOI $=10.1145 / 1746259.1746260$ http://doi.acm.org/10.1145/1746259.1746260

6. Moira Burke and Robert Kraut. 2008. Mopping up: modeling wikipedia promotion decisions. In Proc. of the 2008 ACM conf on Computer supported cooperative work (CSCW '08). ACM, New York, NY, USA, 27-36. http://doi.acm.org/10.1145/1460563.1460571

7. CNN-IBN http://www.ibnlive.com/news/india/how-manyfacebook-users-are-there-in-your-city-485374.html

8. Kate Crawford. 2009. Following you: Disciplines of listening in social media. Continuum: Journal of Media \& Cultural Studies. 23, 4. 525-535. http://doi:10.1080/10304310903003270

9. Kate Crawford. 2011. Listening, not lurking: The neglected for of participation. Cultures of Participation. 63-64.

10. John W. Creswell. 2013. Research design: Qualitative, quantitative, and mixed methods approaches. Sage publications.

11. Judith Donath. 2007. Signals in social supernet. Journal of Computer Mediated Communication. 13, 1. 231-251. http://doi:10.1111/j.1083-6101.2007.00394.x

12. Nicole B. Ellison, Jessica Vitak, Rebecca Gray, and Cliff Lampe. 2014. Cultivating social resources on social network sites: Facebook relationship maintenance behaviors and their role in social capital processes." Journal of ComputerMediated Communication 19, 4. 855-870.

13. Brynn M. Evans and Ed H. Chi. 2008. Towards a model of understanding social search. In Proc. of the 2008 ACM conf on Computer supported cooperative work (CSCW '08). ACM, New York, NY, USA, 485-494.

http://doi.acm.org/10.1145/1460563.1460641

14. Facebook.com/home/story

15. Barney Glaser and Anselm Strauss. 1967. The Discovery of Grounded Theory. Weidenfeld and Nichols

16. Gene Golovchinsky, Meredith Ringel Morris, and Jeremy Pickens. 2010. Editorial: Introduction to the special issue. Information Processing and Management. 46, 6. 629631. http://dx.doi.org/10.1016/j.ipm.2009.11.001

17. Mark S. Granovetter. 1973. The strength of weak ties. American Journal of Sociology. 78, 6. 1360-1380.

18. Anne Oeldorf-Hirsch, Brent Hecht, Meredith Ringel Morris, Jaime Teevan, and Darren Gergle. 2014. To search or to ask: the routing of information needs between traditional search engines and social networks. In Proc. of the Computer supported cooperative work \& social computing (CSCW '14). 16-27. http://doi.acm.org/10.1145/2531602.2531706

19. Maxwell F. Harper, Daniel Moy, and Joseph A. Konstan. 2009. Facts or friends? distinguishing informational and conversational questions in social Q\&A sites. In Proc. of the SIGCHI Conf on Human Factors in Computing Systems (CHI '09). ACM, New York, NY, USA, 759-768. http://doi.acm.org/10.1145/1518701.1518819

20. Bernie Hogan. The presentation of self in the age of social media: Distinguishing performances and exhibitions online. Bulletin of Science, Technology \& Society. http://doi:10.1177/0270467610385893

21. Damon Horowitz and Sepandar D. Kamvar. 2012. Searching the village: models and methods for social search. Commun. ACM 55, 4 (April 2012), 111-118. http://doi.acm.org/10.1145/2133806.2133830

22. Grace YoungJoo Jeon and Soo Young Rieh. 2013. The value of social search: seeking collective personal experience in social Q\&A. In Proc. of the 76th ASIS\&T Annual Meeting: Beyond the Cloud: Rethinking Information Boundaries (ASIST '13), Andrew Grove (Ed.). American Society for Information Science, Silver Springs, MD, USA, Article 7, 10 pages.

23. Angela H. Jiang, Zachary S. Bischof, and Fabian E. Bustamante. 2014. A cliq of content curators. In Proc. of the 2014 ACM conf on SIGCOMM (SIGCOMM '14). ACM, New York, NY, USA, 139-140. http://doi.acm.org/10.1145/2619239.2631470

24. Quentin Jones and Sheizaf Rafaeli. 2000. Time to split, virtually:'Discourse architecture'and'community building' create vibrant virtual publics. Electronic Markets 10, 4. 214223.

25. Mayur Karnik, Ian Oakley, Jayant Venkatanathan, Tasos Spiliotopoulos, and Valentina Nisi. 2013. Uses \& gratifications of a facebook media sharing group. In Proc. of the 2013 conf on Computer supported cooperative work (CSCW '13). ACM, New York, NY, USA, 821-826. http://doi.acm.org/10.1145/2441776.2441868

26. Lee Komito and Jessica Bates. "Virtually local: social media and community among Polish nationals in Dublin." Aslib Proc. Vol. 61. No. 3. Emerald Group Publishing Limited, 2009.

27. Cliff Lampe and Paul Resnick. 2004. Slash(dot) and burn: distributed moderation in a large online conversation space. 
In Proc. of the SIGCHI Conf on Human Factors in Computing Systems (CHI '04). ACM, New York, NY, USA, 543-550. http://doi.acm.org/10.1145/985692.985761

28. Cliff Lampe, Jessica Vitak, Rebecca Gray, and Nicole Ellison. 2012. Perceptions of facebook's value as an information source. In Proc. of the SIGCHI Conf on Human Factors in Computing Systems (CHI '12). ACM, New York, NY, USA, 3195-3204.

http://doi.acm.org/10.1145/2207676.2208739

29. Cliff Lampe, Rebecca Gray, Andrew T. Fiore, and Nicole Ellison. 2014. Help is on the way: patterns of responses to resource requests on facebook. In Proc. of the 17th ACM conf on Computer supported cooperative work \& social computing (CSCW '14). ACM, New York, NY, USA, 3-15. http://doi.acm.org/10.1145/2531602.2531720

30. Jean Lave, and Etienne Wenger. Situated learning: Legitimate peripheral participation. Cambridge university press, 1991.

31. Marwick, A. E., \& Boyd, D. (2011). I tweet honestly, I tweet passionately: Twitter users, context collapse, and the imagined audience. New Media \& Society, 13(1), 114-133.

32. Matthew B. Miles and Michael A. Huberman. 1994. Qualitative Data Analysis: An Expanded Sourcebook: Sage Publications.

33. Meredith Ringel Morris, Jaime Teevan, and Katrina Panovich. 2010. What do people ask their social networks, and why? a survey study of status message q\&a behavior. In Proc. of the SIGCHI Conf on Human Factors in Computing Systems (CHI '10). ACM, New York, NY, USA, 1739-1748. http://doi.acm.org/10.1145/1753326.1753587

34. Meredith Ringel Morris. 2013. Collaborative search revisited. In Proc. of the 2013 conf on Computer supported cooperative work (CSCW '13). ACM, New York, NY, USA, 1181-1192. http://doi.acm.org/10.1145/2441776.2441910

35. Jeffrey Nichols and Jeon-Hyung Kang. 2012. Asking questions of targeted strangers on social networks. In Proc. of the ACM 2012 conf on Computer Supported Cooperative Work (CSCW '12). ACM, New York, NY, USA, 999-1002. http://doi.acm.org/10.1145/2145204.2145352

36. Kenton P. O'Hara, Michael Massimi, Richard Harper, Simon Rubens, and Jessica Morris. 2014. Everyday dwelling with WhatsApp. In Proc. of the 17th ACM conf on Computer supported cooperative work \& social computing (CSCW '14). ACM, New York, NY, USA, 1131-1143. http://doi.acm.org/10.1145/2531602.2531679

37. Namsu Park, Kerk Kee, Sebastian Valenzuela. 2009. Being immersed in social networking environment: Facebook groups, uses and gratifications, and social outcomes. Cyber Psychology \& Behavior. 12, 6. 729-733. http://doi:10.1089/cpb.2009.0003

38. John Postill, and Sarah Pink. 2012. Social media ethnography: the digital researcher in a messy web. Media International Australia

39. Reijo Savolainen. 1995. Everyday life information seeking: approaching information seeking in the context of "way of life". Library \& Information Science Research. 17, 3. 259294.

40. Jeffrey M. Rzeszotarski and Meredith Ringel Morris. 2014. Estimating the social costs of friendsourcing. In Proc. of the SIGCHI Conf on Human Factors in Computing Systems (CHI
'14). ACM, New York, NY, USA, 2735-2744. http://doi.acm.org/10.1145/2556288.2557181

41. Chirag Shah, Jung Sun Oh, and Sanghee Oh. 2008. Exploring characteristics and effects of user participation in online social Q\&A sites. First Monday 13.9.

42. Peter K. Smith, Jess Mahdavi, Manuel Carvalho, Sonja Fisher, Shanette Russell, and Neil Tippett. 2008. Cyberbullying: Its nature and impact in secondary school pupils. Journal of child psychology and psychiatry 49, 4. 376-385.

43. Andrew D. Smock, Nicole B. Ellison, Cliff Lampe, and Donghee Yvette Wohn. 2011. Facebook as a toolkit: A uses and gratification approach to unbundling feature use. Computer Human Behavior. 27, 6 (November 2011), 2322-2329. http://dx.doi.org/10.1016/j.chb.2011.07.011

44. Anselm, Strauss, Juliet M. Corbin. Grounded theory in practice. Sage, 1997

45. The Times of India. Facebook group 'Mums of Bangalore' is the support network for mothers in Bengaluru. November 2015

46. The Times of India. Bangalore gets lion's share of educated migrants. February 2013

47. Alex S. Taylor, Siân Lindley, Tim Regan, David Sweeney, Vasillis Vlachokyriakos, Lillie Grainger, and Jessica Lingel. 2015. Data-in-Place: Thinking through the Relations Between Data and Community. In Proc. of the 33rd Annual ACM Conf on Human Factors in Computing Systems (CHI '15). ACM, New York, NY, USA, 2863-2872. http://doi.acm.org/10.1145/2702123.2702558

48. Mike Thelwall and David Wilkinson. 2010. Public dialogs in social network sites: what is their purpose? Journal of the American Society for Information Science and Technology. 61, 2. 392-404. http://doi:10.1002/asi.21241

49. Nigel Thrift. 2014. The sentient city and what it may portend. Big Data \& Society. 1.1. http://doi:10.1177/2053951714532241

50. Zeynep Tufekci. 2008. Grooming, gossip, Facebook and MySpace: what can we learn about these sites from those who won't assimilate? Information, Communication \& Society. 11. 4. 544-564. http://doi:10.1080/13691180801999050

51. Jessica Vitak and Nicole B. Ellison. 2012. 'There's a network out there you might as well tap' Exploring the benefits of and barriers to exchanging informational and support-based resources on Facebook. New Media \& Society 15, 2: 243259. http://doi/10.1177/1461444812451566

52. Andreas Wittel. 2001. Toward a network sociality. Theory, Culture \& Society. 18, 6. 51-76. http://doi:10.1177/026327601018006003

53. Donghee Yvette Wohn, Cliff Lampe, Jessica Vitak, and Nicole B. Ellison. 2011. Coordinating the ordinary: social information uses of Facebook by adults. In Proc. of the 2011 iConf(iConf '11). ACM, New York, NY, USA, 340-347. http://doi.acm.org/10.1145/1940761.1940808

54. Zhe Zhao and Qiaozhu Mei. 2013. Questions about questions: an empirical analysis of information needs on Twitter. In Proc. of the 22nd international conf on World Wide Web (WWW '13). International World Wide Web Confs Steering Committee, Republic and Canton of Geneva, Switzerland, 1545-1556. 